\title{
Research on Unmanned Airborne Millimeter - wave Broadband Obstacle Avoidance Radar System Based on FPGA
}

\author{
Yiran $\mathrm{Ma}^{1, \mathrm{a},{ }^{*}}$, Wei $\mathrm{Xu}^{2, \mathrm{~b}}$, Wanguo Wang ${ }^{2, \mathrm{c}}$, Liang $\mathrm{Liu}^{2, \mathrm{~d}}$ \\ ${ }^{1}$ Shandong University of Science and Technology, Jinan 250031, China \\ 2Shandong Luneng Intelligence Technology Co., Ltd. Jinan, 250101, China \\ aLixiangping401@126.com, blzyydhcgd@163.com, cwangwanguo03@qq.com, \\ dmsxnjust@139.com
}

Keywords: Millimeter wave obstacle avoidance radar, linear frequency modulation source, FPGA.

\begin{abstract}
UAVs often need to perform tasks in low-level complex environments. In order to protect the safety of UAVs, it is necessary to avoid radar and to avoid early warning and obstruction. In this paper, the obstacle avoidance radar of UAV is analyzed deeply, and a stable, low cost and miniaturized LFM broadband millimeter wave obstacle avoidance radar is designed to solve the problem of UAV flight safety. The test data show that the designed millimeter wave obstacle avoidance radar system meets the requirements of UAV obstacle avoidance.
\end{abstract}

\section{Introduction}

UAV obstacle avoidance generally use the technology: optical detectors, infrared detectors, laser radar and millimeter-wave radar and other technologies ${ }^{[1-7]}$. In the fog, strong sun exposure and other harsh conditions, visual detection and infrared detection basically lost its role ${ }^{[8]}$. The lidar is too large to be used on unmanned aerial vehicles. Millimeter-wave radar obstacle avoidance technology is the most potential, this is because the ability to load unmanned aircraft is limited, in order to maximize the implementation of the task, the size and weight of airborne equipment should be as little as possible, millimeter-wave radar obstacle avoidance Technology just to meet this requirement ${ }^{[9-12]}$. Millimeter-wave radar with a large bandwidth, narrow beam, low wavelength, low power consumption advantages, fully meet the requirements, millimeter-wave radar can rain and snow, haze, dust and other bad weather under normal work, with a strong anti-Clutter capability, so that UAV can work around the clock. Therefore, millimeter-wave radar obstacle avoidance technology is an optimal choice for UAV obstacle avoidance system ${ }^{[13-16]}$. All manuscripts must be in English, also the table and figure texts, otherwise we cannot publish your paper. Please keep a second copy of your manuscript in your office. When receiving the paper, we assume that the corresponding authors grant us the copyright to use the paper for the book or journal in question. Should authors use tables or figures from other Publications, they must ask the corresponding publishers to grant them the right to publish this material in their paper.

\section{Millimeter Wave Unmanned Aerial Vehicle Obstacle Avoidance Radar System}

Obstacle avoidance system has three main components: transmitter, receiver front and signal processing module. The system block diagram is shown in Fig. 1. The transmitter module consists of a VCO, a power divider, a frequency multiplier and an amplifier. The main function is to generate a high frequency signal and amplify the signal through the isolator and finally through the antenna. Receiver mainly by the mixer, low noise, IF amplification and filter circuit, the main function is to receive the echo signal, and the signal amplification, mixing and filtering for the follow-up signal processing to prepare. Signal processing module is mainly implemented on the FPGA, the main function is to deal with the front of the receiver signal to do digital processing, and ultimately the distance information extracted from the target, and through the serial module sent to the ground control station, ground control station control People can make real-time responses. 


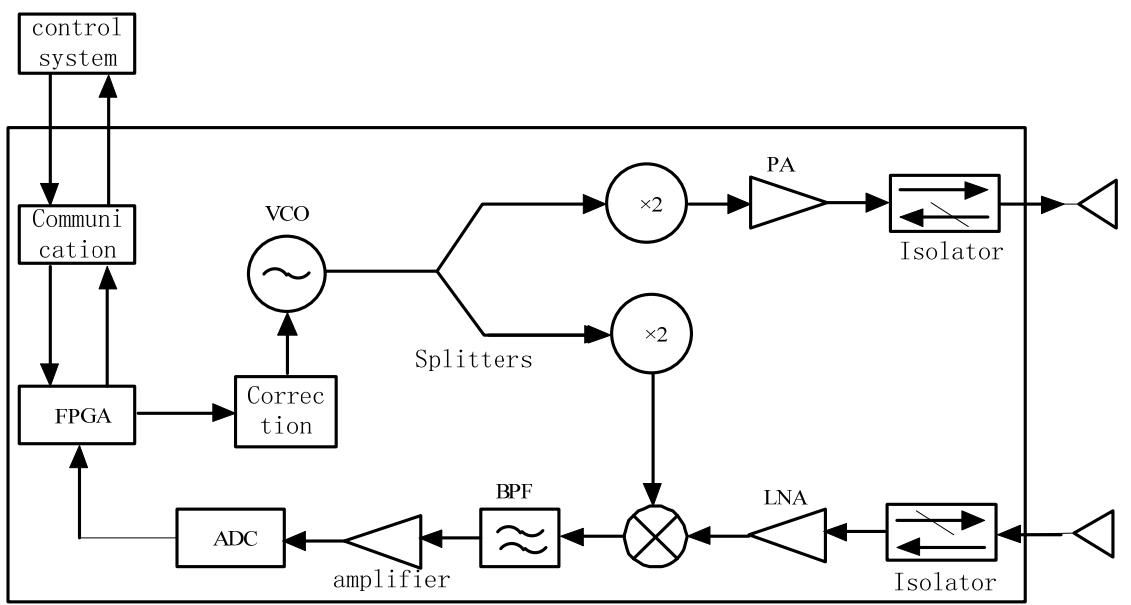

Fig. 1 block diagram of obstacle avoidance system

\subsection{Millimeter Wave Broadband Linear Frequency Modulation Avoidance Radar Transmitter}

According to the transmitter index and radar system requirements, the transmitter diagram shown in Fig. 2. First through the FPGA linear frequency modulation VCO, resulting in $18.8-19 \mathrm{GHz}$ linear FM signal, through the frequency multiplier to 37.6-38GHz linear FM signal, amplified by the power amplifier, and finally through the antenna radiation out. The program is simple, economic, volume and power consumption are smaller.

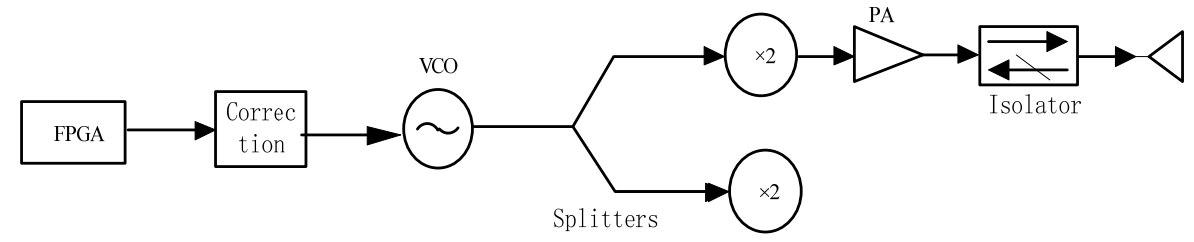

Fig. 2 Schematic diagram of the transmitter

\subsection{Front - end design of millimeter - wave broadband LFM radar receiver}

The block diagram of the zero-IF receiver is shown in Fig. 3. The received RF signal is amplified by the low noise amplifier and mixed with the local oscillator signal. Since the local oscillation signal and the frequency and the frequency of the RF signal are the same, the frequency signal close to zero frequency is directly generated after mixing, and then a low frequency intermediate frequency The signal processing unit performs filtering and amplification. In order to facilitate the next stage of FPGA signal processing. Because this program is simple, high reliability, can meet the requirements of the indicators at the same time in terms of volume and power consumption can be smaller.

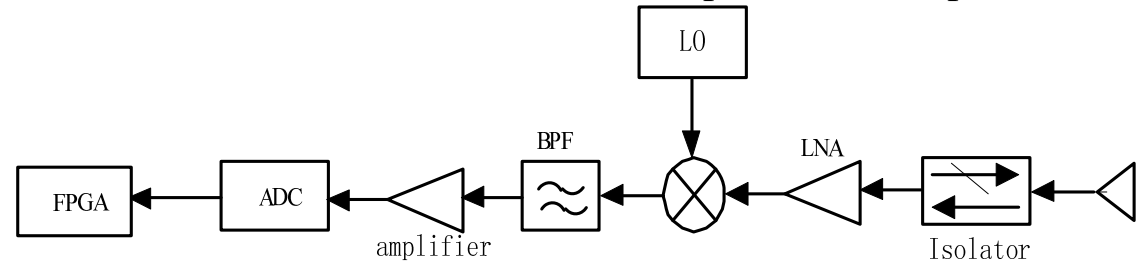

Fig. 3 Block diagram of the receiver

\subsection{Signal Processing Module Based on FPGA}

The application of this project is mainly small unmanned aerial vehicles to obstruct the power line, small UAV in flight through other auxiliary equipment can make small unmanned aerial vehicles and power lines in a plane, so the state of the power line The intensity of the scattering is relatively strong, the accumulation of time does not need to be too long, there is a reason because the small UAV flight speed is slower, the detection distance is short, the ground strong clutter in space and frequency domain filter filter, The complexity of this system can greatly reduce the complexity of the signal processing, the above reasons, FPGA-based signal processing program is: A / D module to collect the beat signal, the collected digital signal fast Fourier transform, non-coherent accumulation, And then do constant false alarm processing, and finally output distance information. 


\section{Realization of Obstacle Avoidance Radar System and Analysis of Measured Data}

This radar system is designed for unmanned aerial vehicle power line obstacle avoidance. The radar system includes radar transmitter, radar receiver, signal processing module and antenna. The physical map is shown in Fig. 4. In Shandong, a test site carried out the field flying test, the radar installation diagram is shown in Fig. 5.

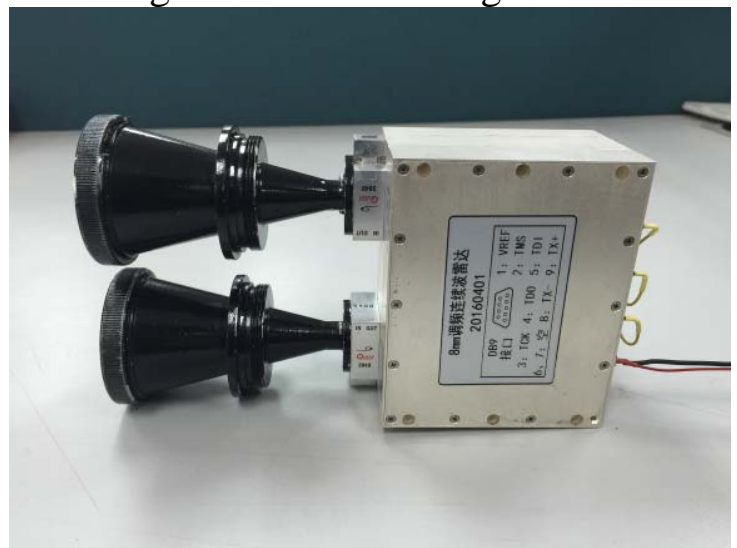

Fig. 4 Obstacle avoidance physical map

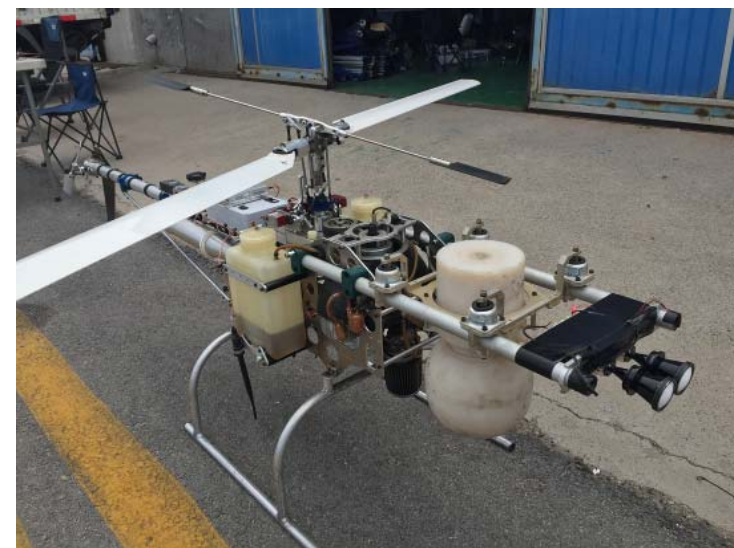

Fig. 5 Field test device diagram

When the UAV is flying in the air, the millimeter wave obstacle avoidance radar sends the obstacle distance information detected by the front through the serial module to the airborne station and forwards the distance information of the obstacle to the ground control station via the airborne radio station and According to the information returned, the ground station control personnel on the flight path and flight status of the aircraft in real time to adjust, in the field fly test, the ground station control personnel can be hikes such as hover, flight, turn, Rise and fall and other operations. When the unmanned aerial vehicles in front of a certain range of obstacles, the ground station will be red "alarm" words. Obstacle avoidance system status code is $0 x 01$, indicating that the obstacle avoidance system is on, as shown in Fig. 6. Obstacle avoidance system status code is 0x00, indicating that the obstacle avoidance system is off state. As shown in Fig. 7.

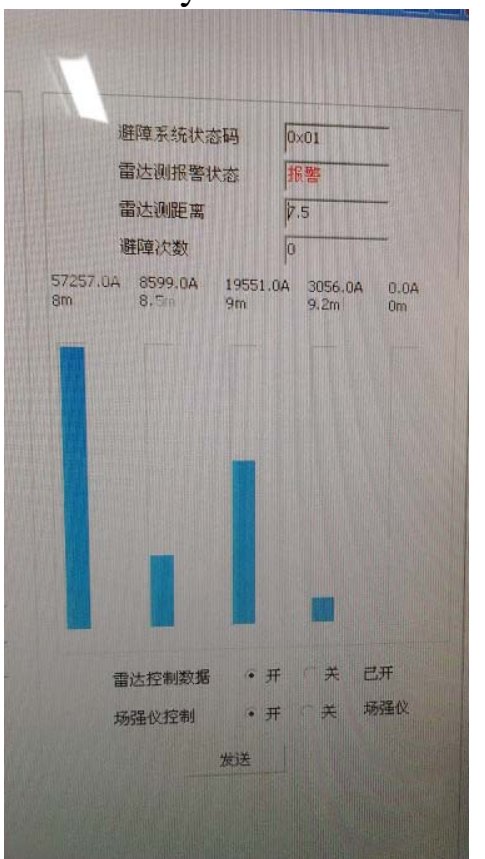

Fig.6 Ground control station displays information

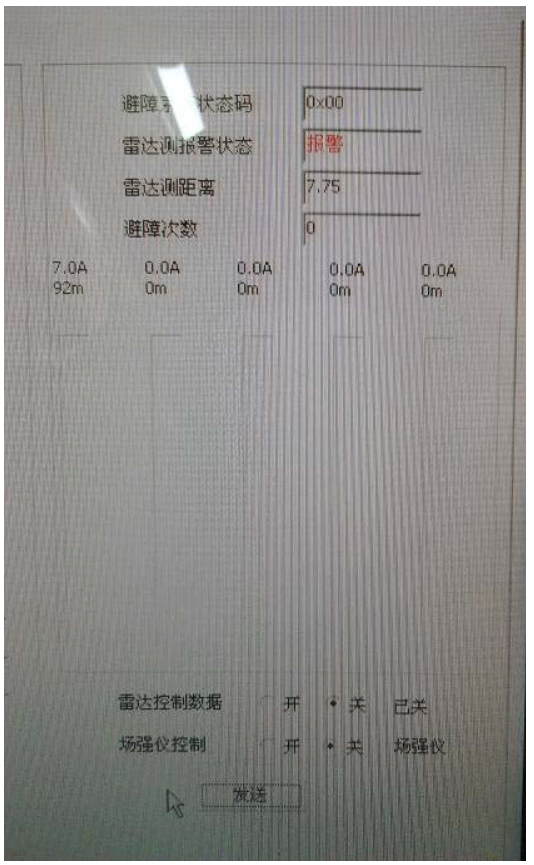

Fig. 7 Obstacle Avoidance System Off State Ground Station Display 


\section{Concluding remarks}

In order to protect the safety of UAV operations, the need to avoid radar for early warning and obstacle avoidance. The analysis of UAV obstacle avoidance radar is carried out, and a stable, low cost and miniaturized LFM broadband millimeter wave obstacle avoidance radar is designed. From the above field simulation experimental data show that the system can detect unmanned aerial vehicles in front of obstacles (including power lines) to meet the system requirements.

\section{References}

[1] SHEN Tao, SUN Zhong-liang.Study on linear correction scheme of millimeter-wave VCO electrical characteristics [J]. Modern Radar, 1997, 19 (1): 91-100.

[2] SHEN Tao, SUN Zhong-liang. Development of $35 \mathrm{GHz}$ High Linear VCO [J]. Journal of Southeast University, 1996, 26 (1): 14-18.

[3] Jin-xin, XU Jian-zhong. Ka-band VCO design and closed-loop linear correction [D]. Nanjing: Nanjing University of Science and Technology, 2007.

[4] LENG Jian-wei, QI Xiao-hui.Study on linear correction technique of VCO electrical characteristics [J]. Microelectronics, 2012, 42 (3): 440-444.

[5] JIANG Xing, LIU Yao-shan, SUN Feng-yan.A linear correction method for vehicle-mounted radar voltage-controlled oscillator [J]. Journal of Radio Science, 2013, 28 (2): 368-371.

[6] Journal of Systems Engineering and Electronics, 2001, 23 (2): 16-18. ZHAO Zhi-ming, DING Yi-yuan, YUAN Jing-cheng. Relative Nonlinear Correction Method for Broadband LFMCW Sweep Source $[\mathrm{J}]$.

[7] Blaser R J. Analogmultipllers drive component linerlzation scheme [J]. Microwave\&RF, 1992, 31(10): 74-80.

[8] Yan G W, Xie G, Qiu Y X, et al. MEA based nonlinearity correction algorithm for the VCO of LFMCW radar level guage[C]. 10th International Conference, RSFDGrC 2005 Regina, Canada, 2005: 460-470.

[9] Kwon H, Kang B. Linear frequency modulation of voltage-controlled oscillator using delay-line feedback [J]. IEEE Microwave Wireless Compon Lett, 2005, 15(6): 120-123.

[10]Li Feng, Guo Dechun. Using VCO to Realize Variable Frequency Diode Direct Frequency Modulation [J]. Today Electronics, 2005, 1 (3): 63-64.

[11] Kim C W, Koo K H, Yoon S W. Fully integrated wideband CMOS VCO with improved f-V linearity and low tuning [J]. Elec Lett, 2010, 46(1): 29-30.

[12] Colodro F, Torralba A. Linearity Enhancement of VCO-Based Quantizers for SD Modulators by Means of a Tracking Loop [J]. Circuits \& Systems II Express Briefs IEEE Transactions on, 2014, 61(6): 383-387.

[13]Bai X, Zhu Z, Su L, et al. A Wide Tuning Range and High Linearity VCO Circuit Design [C]. Computer Science and Electronics Engineering (ICCSEE), 2012 International Conference on. IEEE, 2012: 208-211.

[14]El-Halwagy W, Mohamed Dessouky, El-Ghitani H. A programmable 8-bit, 10MHz BW, 6.8mW, 200MSample/sec, 70dB SNDR VCO-based ADC using SC feedback for VCO linearization [C]// IEEE, International Conference on Electronics, Circuits, and Systems. IEEE, 2013: 157-160.

[15] AadilRafeeque K P, Sahu A. A cost effective static linearity testing scheme for ADCs[C]. Electrical, Computer and Communication Technologies (ICECCT), 2015 IEEE International Conference on. IEEE, 2015: 1-4.

[16] Johnson W. Digitally Tuned PROM-Corrected VCO [J]. Microwave Journal, 1982, 25(11): 131-132. 